\title{
Supporting Information for Cartesian Decomposition of Infrared Spectra Reveals the Structure of Solution- Deposited, Self-Assembled Benzoate and Alkanoate Monolayers on Rutile (110)
}

\author{
William J. I. DeBenedetti, Erik S. Skibinski, Joshua A. Hinckley, \\ Sara B. Nedessa, and Melissa A. Hines* \\ Dept. of Chemistry and Chemical Biology, Cornell University, Ithaca NY 14853 USA
}

\section{Infrared Spectra of Heptanoate Monolayers on Rutile (110)}

The spectra of heptanoate monolayers on rutile (110) were very similar to those obtained from octanoate monolayers (except for a small intensity decrease), as shown in Figure $\mathrm{S} 1$. This similarity is noteworthy because of the structural differences between the all-trans conformers of octanoate and heptanoate. As shown by Figure 8, the transition dipole moment of the symmetric $\mathrm{CH}_{3}$ stretch is vertically polarized for the all-trans conformer of octanoate. In contrast, the corresponding dipole moment in heptanoate is horizontally polarized. The fact that this change is not apparent in comparing Figure 6 and Figure S 1 provides additional evidence for a high density of gauche conformers.

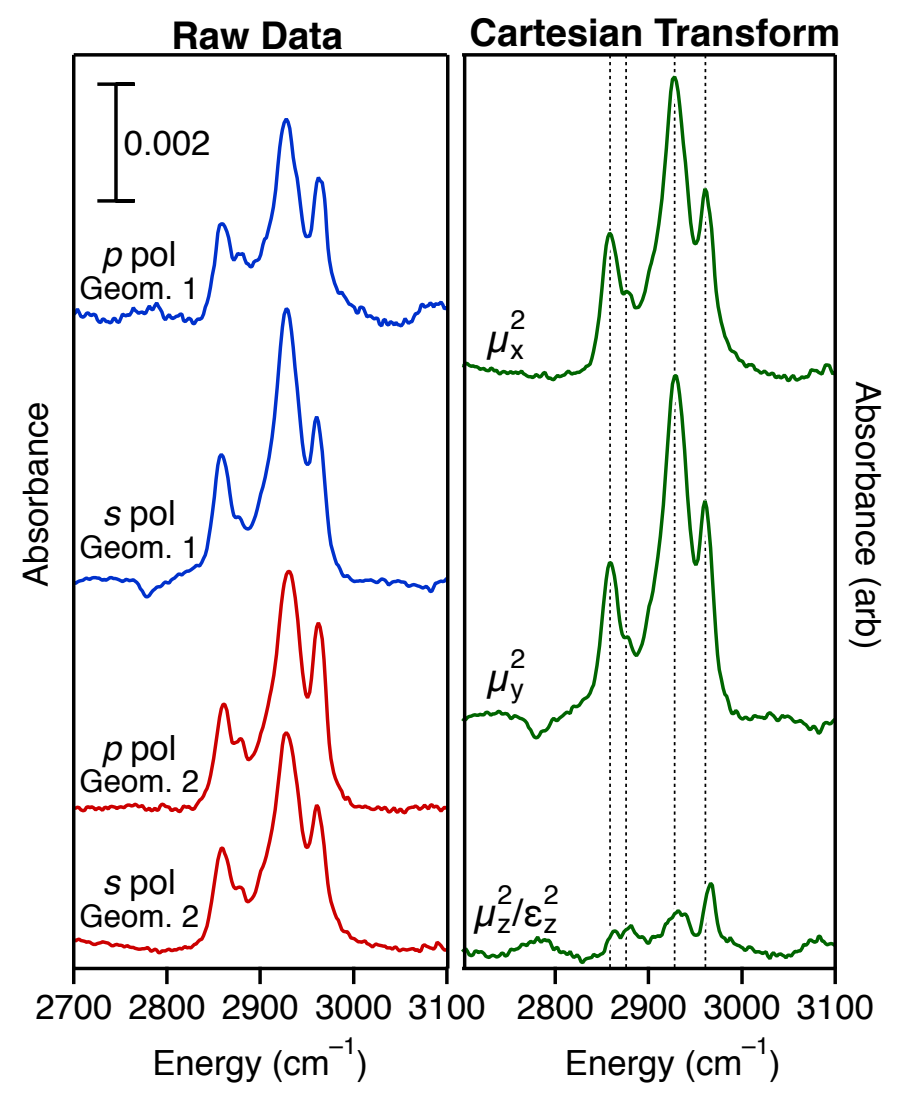

Figure S 1: (Left) The $\mathrm{C}-\mathrm{H}$ stretch region of four infrared spectra of heptanoate $\left(\mathrm{C}_{6} \mathrm{H}_{13} \mathrm{COO} / \mathrm{TiO}_{2}\right)$ monolayers obtained in the geometries defined in Figure 1. (Right) Cartesian transform of the four experimental spectra revealed the transition dipole moment projected along the $x$ (Ti row or [001] direction), $y$ ([1 $\overline{1} 0]$ direction), and $z$ axes. The dotted lines represent the transitions in Table 3. 


\section{XPS Quantification of Heptanoate Coverage}

The monolayer coverage $\phi$ of heptanoate was calculated from the ratio of the integrated intensities of the $\mathrm{C} 1 s$ and Ti $2 p$ transitions, $I_{C}$ and $I_{T i}$, using the Beer-Lambert Law and the sample geometry. The height of the heptanoate layer, $a$, was estimated to be $0.68 \mathrm{~nm}$ from the DFT simulation. Similarly, the relative densities of $C$ in the heptanoate layer to that of graphite (the $C$ standard), $\varrho_{r}$, was found to be 0.232 . To account for the lower probability of electron scattering in the heptanoate monolayer compared to graphite, the inelastic mean free path of $\mathrm{C} 1 s$ photoelectrons, $\lambda_{\mathrm{C}}$, was multiplied by $\mathrm{Q}_{\mathrm{r}}{ }^{-1}$. Photoelectrons were collected at angle $\theta$ from the surface normal. The relative intensities the $\mathrm{C} 1 s$ transition to Ti $2 p$ transition was therefore given by

$$
\frac{I_{C}}{I_{T i}}=\frac{\rho_{r}}{0.333} \frac{\Gamma_{C}}{\Gamma_{T i}} \frac{\phi \int_{0}^{a} e^{-x \rho, / \lambda_{c} \cos \theta} d x}{\int_{0}^{\infty} e^{-a \rho_{r} / \lambda_{n} \cos \theta} e^{-x / \lambda_{n} \cos \theta} d x},
$$

where $\Gamma_{\mathrm{C}}$ and $\Gamma_{\mathrm{T} i}$ are the relative photoemission cross sections for $\mathrm{C} 1 s$ and Ti $2 p$. The factor of $1 / 3$ accounts for the stoichiometry of $\mathrm{TiO}_{2}$, and $\lambda_{\mathrm{Ti}}$ is the mean free path of photoelectrons in $\mathrm{TiO}_{2}$. Expanding the integrals in Eqn. (1) to first order and simplifying yielded

$$
\frac{I_{C}}{I_{T i}}=\frac{\rho_{r}}{0.333} \frac{\Gamma_{C}}{\Gamma_{T i}} \frac{\phi a}{e^{-a \rho_{r} / \lambda_{n} \cos \theta} \lambda_{T i} \cos \theta} .
$$

The ratio of $\mathrm{C} 1 s$ to Ti $2 p$ from pure samples of graphite and titanium was given by

$$
\frac{I_{C}^{\infty}}{I_{T i}^{\infty}}=\frac{\Gamma_{C}}{\Gamma_{T i}} \frac{\int_{0}^{\infty} e^{-x / \lambda_{c} \cos \theta} d x}{\int_{0}^{\infty} e^{-x / \lambda_{n} \cos \theta} d x}=\frac{\Gamma_{C}}{\Gamma_{T i}} \frac{\lambda_{C}}{\lambda_{T i}}=\frac{R S F_{C}}{R S F_{T i}},
$$

where $\mathrm{RSF}_{\mathrm{Ti}} / \mathrm{RSF}_{\mathrm{C}}$ was the ratio of relative sensitivity factors for Ti $2 p$ and $\mathrm{C} 1 s$ and was equal to 7.2. ${ }^{1}$ Combining Eqn. (2) and Eqn. (3) and using $\lambda_{\mathrm{C}}=1.54 \mathrm{~nm}$ and $\lambda_{\mathrm{T} i}=1.27 \mathrm{~nm}$ from Ref. 2 gave

$$
\phi=23.4 e^{-0.124 / \cos \theta} \cos \theta \frac{I_{C}}{I_{T i}} .
$$

\section{Structures used in DFT Calculations}

Structures were modeled using $2 \times 2$ and $4 \times 2$ periodically repeating slabs, where the first and second integers refer to the number of repeat units in the [001] and [1 10$]$ directions, respectively, consisting of 5 $\mathrm{TiO}_{2}$ trilayers separated by a $12.5 \AA$ vacuum spacing. A $4 \times 2 \times 1$ and a $2 \times 2 \times 1$ Monkhorst-Pack grid of $k$ points was used, respectively.

The slab used to model the paired tetramer configuration is shown in Figure $\mathrm{S} 2$, whereas the slab used to model the twisted monomer is shown in Figure S 3. The DFT simulations predicted that the twisted monomer configuration was $0.60 \mathrm{meV} /$ benzoate molecule more stable than the tilted tetramer configuration. In contrast, the experiments showed that the tilted tetramer configuration was approximately $22 \mathrm{meV} /$ benzoate molecule more stable than the twisted monomer configuration. 


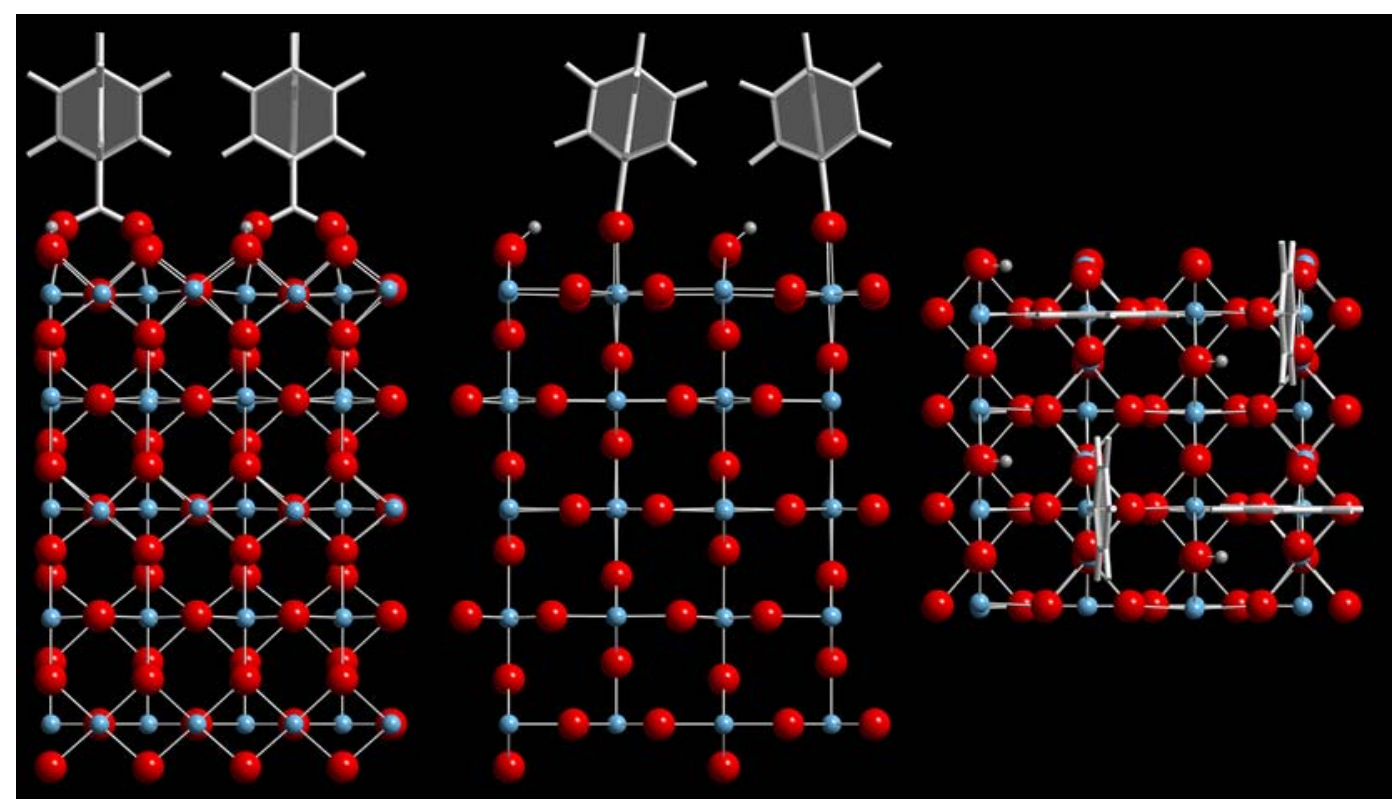

Figure S 2: Structure of the relaxed supercell used to represent the tilted tetramer isomer of a benzoate monolayer. The views are from the side along the (left) [1 $\overline{1} 0]$ and (middle) [001] directions, and from the (right) top. The red, white, and blue balls represent $\mathrm{O}, \mathrm{H}$, and $\mathrm{Ti}$ atoms, respectively.

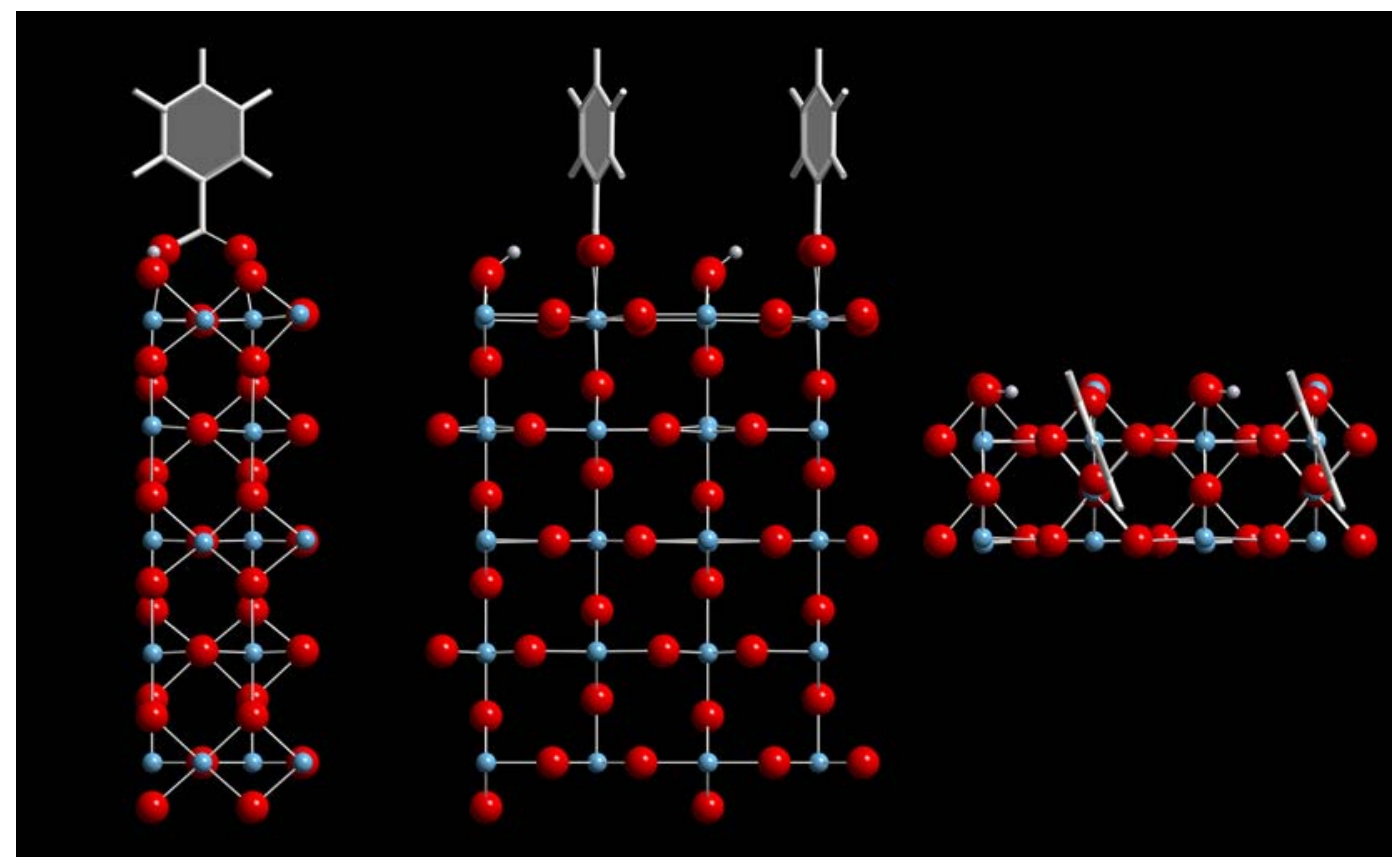

Figure S 3: Structure of the relaxed supercell used to represent the twisted monomer isomer of a benzoate monolayer. The views are from the side along the (left) [1 $\overline{1} 0]$ and (middle) [001] directions, and from the (right) top. The red, white, and blue balls represent $\mathrm{O}, \mathrm{H}$, and $\mathrm{Ti}$ atoms, respectively. 


\section{Code for Cartesian Decomposition}

The following code can be used directly in Igor Pro ${ }^{3}$ to perform Cartesian decompositions of experimental spectra taken on rutile (110) surfaces. To use this code with a different uniaxial substrate, the function SetupDielectric must be modified to contain the correct, wavelength dependent dielectric constants. The code can be easily adapted to any C-like language with minor modifications.

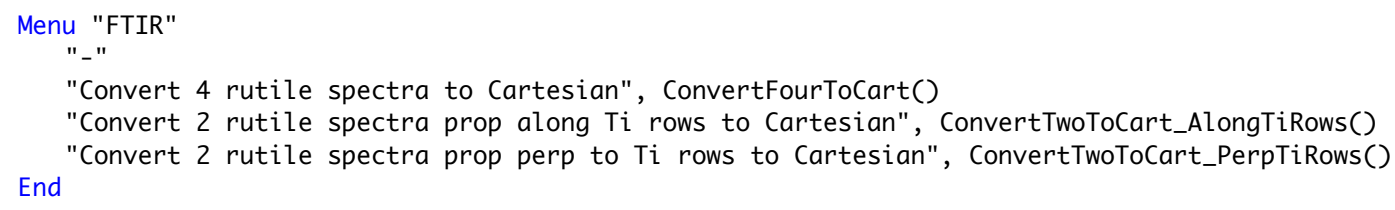

// This function calculates the $\mathrm{x}-, \mathrm{y}-$, and $\mathrm{z}$-components of a surface spectrum from s- and p-polarized

// spectra taken in two orthogonal geometries. The $x$ direction is along the Ti rows, the $y$ direction is perpendicular.

Proc ConvertFourToCart(sAp1, sAs1, sAp2, sAs2)

string sAp1, sAs1, sAp2, sAs2

Prompt sAp1,"P-pol spectrum propagating along Ti rows", popup, WaveList("*", ";", "")

Prompt sAs1,"S-pol spectrum propagating along Ti rows",popup, WaveList("*", ";"," ")

Prompt sAp2,"P-pol spectrum propagating perp to Ti rows",popup, WaveList("*", ";"," ")

Prompt sAs2,"S-pol spectrum propagating perp to Ti rows",popup, WaveList("*", ";", "")

End

doConvertFourToCart(\$sAp1, \$sAs1, \$sAp2, \$sAs2)

$/ /$ This function calculates the Cartesian components of a surface spectrum from four experimental spectra.
$/ /$ Inputs: Four waves (surface absorbances: Ap1, As1, Ap2, As2) as a function of energy in $\mathrm{cm}-1$
$/ / \quad$ Outputs: Three waves corresponding to the Cartesian components of the surface spectrum.
$/ / \quad$ mu2_row corresponds to the in-plane component aligned with the Ti rows (i.e., $x$ axis)
$/ / \quad$ mu2_cross corresponds to the in-plane component perpendicular to the Ti rows (i.e., y axis)
$/ / \quad$ mu2_z corresponds to the component perpendicular to the surface (i.e., z axis)
Function doConvertFourToCart(Ap1, As1, Ap2, As2)
wave Ap1, As1, Ap2, As2

SetupDielectric()

duplicate/o Ap1, mu2_row, mu2_cross, mu2_z, denom

denom $=\operatorname{Ill}(e e(x))^{\wedge} 2 * \operatorname{Ir}(e o(x))^{\wedge} 2 * \operatorname{Is}(e o(x))^{\wedge} 2+\left(\operatorname{Ill}(e o(x))^{\wedge} 2 * \operatorname{Ir}(e e(x))^{\wedge} 2+\left(\operatorname{Ir}(e e(x))^{\wedge} 2+\operatorname{Ir}(e o(x))^{\wedge} 2\right)^{*} \operatorname{Is}(e o(x))^{\wedge} 2\right)^{*} \operatorname{Is}(e p(x))^{\wedge} 2$

mu2_row $=\operatorname{Ill}(e e(x)) * \operatorname{Ir}(e o(x)) * \operatorname{Is}(e o(x)) *(\operatorname{As} 1 * \operatorname{Ill}(e o(x)) * \operatorname{Ir}(e e(x))+(-\operatorname{Ap2} * \operatorname{Ir}(e e(x))+e t a(x) * A p 1 * \operatorname{Ir}(e o(x))) * \operatorname{Is}(e o(x)))$ mu2_row $+=\operatorname{As} 2 *\left(\operatorname{Ill}(e o(x))^{\wedge} 2 * \operatorname{Ir}(e e(x))^{\wedge} 2+\left(\operatorname{Ir}(e e(x))^{\wedge} 2+\operatorname{Ir}(e o(x))^{\wedge} 2\right) * \operatorname{Is}(e o(x))^{\wedge} 2\right)^{*} \operatorname{Is}(e p(x))$

mu2_row $/=$ denom

mu2_cross $=\operatorname{Ill}($ eo $(x)) * \operatorname{Ir}(e e(x)) * \operatorname{Is}(e p(x)) *(\operatorname{As} 2 * \operatorname{Ill}(e e(x)) * \operatorname{Ir}(e o(x))+(\operatorname{Ap} 2 * \operatorname{Ir}(\operatorname{ee}(x))-e t a(x) * A p 1 * \operatorname{Ir}(\operatorname{eo}(x))) * \operatorname{Is}(\operatorname{ep}(x)))$

mu2_cross $+=\operatorname{As} 1 * \operatorname{Is}(e o(x)) *\left(\operatorname{Ill}(e e(x))^{\wedge} 2 * \operatorname{Ir}(e o(x))^{\wedge} 2+\left(\operatorname{Ir}(e e(x))^{\wedge} 2+\operatorname{Ir}(e o(x))^{\wedge} 2\right) * \operatorname{Is}(e p(x))^{\wedge} 2\right)$

mu2_cross /= denom

mu2_z $=\operatorname{Ir}(e e(x)) *(\operatorname{Ill}(e o(x)) \wedge 2+\operatorname{Is}(e o(x)) \wedge 2) * \operatorname{Is}(e p(x)) *(-\operatorname{As} 2 * \operatorname{Ill}(e e(x))+e t a(x) * A p 1 * \operatorname{Is}(e p(x)))$

mu2_z $=\operatorname{As} 1 * \operatorname{Ill}(\mathrm{eo}(\mathrm{x})) * \operatorname{Ir}(\mathrm{eo}(\mathrm{x})) * \operatorname{Is}(\operatorname{Eo}(x)) *\left(\operatorname{Ill}(\mathrm{ee}(\mathrm{x}))^{\wedge} 2+\operatorname{Is}(\mathrm{ep}(\mathrm{x}))^{\wedge} 2\right)$

mu2_z $+=\operatorname{Ap} 2 * \operatorname{Ir}(e o(x)) * \operatorname{Is}(e o(x)) \wedge 2 *(\operatorname{Ill}(e e(x)) \wedge 2+\operatorname{Is}(e p(x)) \wedge 2)$

mu2_z /= denom

End

KillWaves/Z cm1_dat, eo_dat, ep_dat, denom

// This function calculates the in-plane- and z-components of a surface spectrum from s-and p-polarized

// spectra taken with light propagating along the Ti rows (i.e., in the plane of the optic axis)

// assuming the spectrum has sufficient symmetry to lead to identical $x$ - and $y$ - polarized spectra.

Proc ConvertTwoToCart_AlongTiRows(Ppol, Spol)

string Ppol, Spol

Prompt Ppol, "P-polarized spectrum:",popup, WaveList("*", ";","")

Prompt Spol, "S-polarized spectrum:",popup, WaveList("*", ";","")

SetupDielectric()

duplicate/o \$Ppol, mu2_inplane, mu2_z

mu2_inplane $=\$$ Spol $/ \operatorname{Is}(e o(x))$

mu2_z $=($ eta $(x) * \$ P p o l * \operatorname{Is}(e o(x))-\$ \operatorname{Spol} * \operatorname{Ill}(\operatorname{ee}(x))) /(\operatorname{Ir}(\operatorname{ee}(x)) * \operatorname{Is}(\operatorname{eo}(x)))$ 
KillWaves/Z cm1_dat, eo_dat, ep_dat

End

// This function calculates the in-plane- and z-components of a surface spectrum from s-and p-polarized

// spectra taken with light propagating perpendicularly to the Ti rows (i.e., in the plane of the optic axis)

// assuming the spectrum has sufficient symmetry to lead to identical $x$ - and $y$-polarized spectra.

Proc ConvertTwoToCart_PerpTiRows(Ppol, Spol)

string Ppol, Spol

Prompt Ppol, "P-polarized spectrum:", popup, WaveList("*", ";"," ")

Prompt Spol, "S-polarized spectrum:",popup, WaveList("*", ";","")

SetupDielectric()

duplicate/o \$Ppol, mu2_z, mu2_inplane

mu2_inplane $=\$$ Spol $/ \operatorname{Is}(\mathrm{ep}(\mathrm{x}))$

mu2_z $=(\$ \operatorname{Ppol} * \operatorname{Is}(\mathrm{ep}(\mathrm{x}))-\$ \operatorname{Spol} * \operatorname{Ill}(\mathrm{eo}(\mathrm{x}))) /(\operatorname{Ir}(\operatorname{eo}(\mathrm{x})) * \operatorname{Is}(\operatorname{ep}(\mathrm{x})))$

KillWaves/Z cm1_dat, eo_dat, ep_dat

End

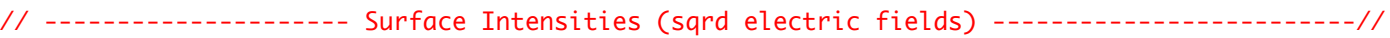

// Surface Elec Field squared (two-layer model) from p. 223 of Y. J. Chabal, Surf. Sci. Reports,

// 8, 211-357 (1988). Note that s-pol light is parallel to the y-direction, not the x-direction.

Function Ill(eps) // I parallel; Equiv to Ex^2; eps = epsilon of substrate

variable eps

variable num, denom, invEps

invEps $=1 /$ eps

num $=4 * \operatorname{CosD}(45) \wedge 2 *(\operatorname{SinD}(45) \wedge 2-$ invEps $)$

denom $=(1-$ invEps $) *((1+$ invEps $) * \operatorname{SinD}(45) \wedge 2-$ invEps $)$

End

return num/denom

Function Is(eps) // Is; Equiv to Ey^2; eps = epsilon of substrate

variable eps

return $4 * \operatorname{CosD}(45)^{\wedge} 2 /(1-1 /$ eps $)$

End

Function $\operatorname{Ir}(\mathrm{eps}) / /$ I perp; Equiv to Ez^2; eps = epsilon of substrate

variable eps

variable num, denom, invEps

invEps $=1$ eps

num $=(2 * \cos D(45) * \operatorname{sinD}(45))^{\wedge} 2$

denom $=(1-$ invEps $) *\left((1+\right.$ invEps $) * \operatorname{sinD}(45)^{\wedge} 2-$ invEps $)$

End

return num/denom

Function SetUpDielectric()

// Rutile data from Palik, Handbook of Optical Constants of Solids, pp. 795-804.

// eo_dat is n_perp^2; ep_dat is n_para^2

make/n=8/o cm1_dat, eo_dat, ep_dat

$\mathrm{cm1}$ dat $=\{4133,4132,2959,2639,2336,2045,1745,1744\}$

eo_dat $=\{2.4,2.4,2.41,2.39,2.34,2.32,2.24,2.24\}$

ep_dat $=\{2.59,2.59,2.58,2.57,2.51,2.49,2.43,2.43\}$

End

eo_dat ${ }^{*}=$ eo_dat; ep_dat ${ }^{*}=$ ep_dat

Function eo $(\mathrm{cm} 1)$

variable $\mathrm{cm} 1$

// Return the dielectric constant for the ordinary wave, eps_perp, at a specific energy in $\mathrm{cm}-1$

wave cm1_dat, eo_dat

End

return interp(cm1, cm1_dat, eo_dat) 


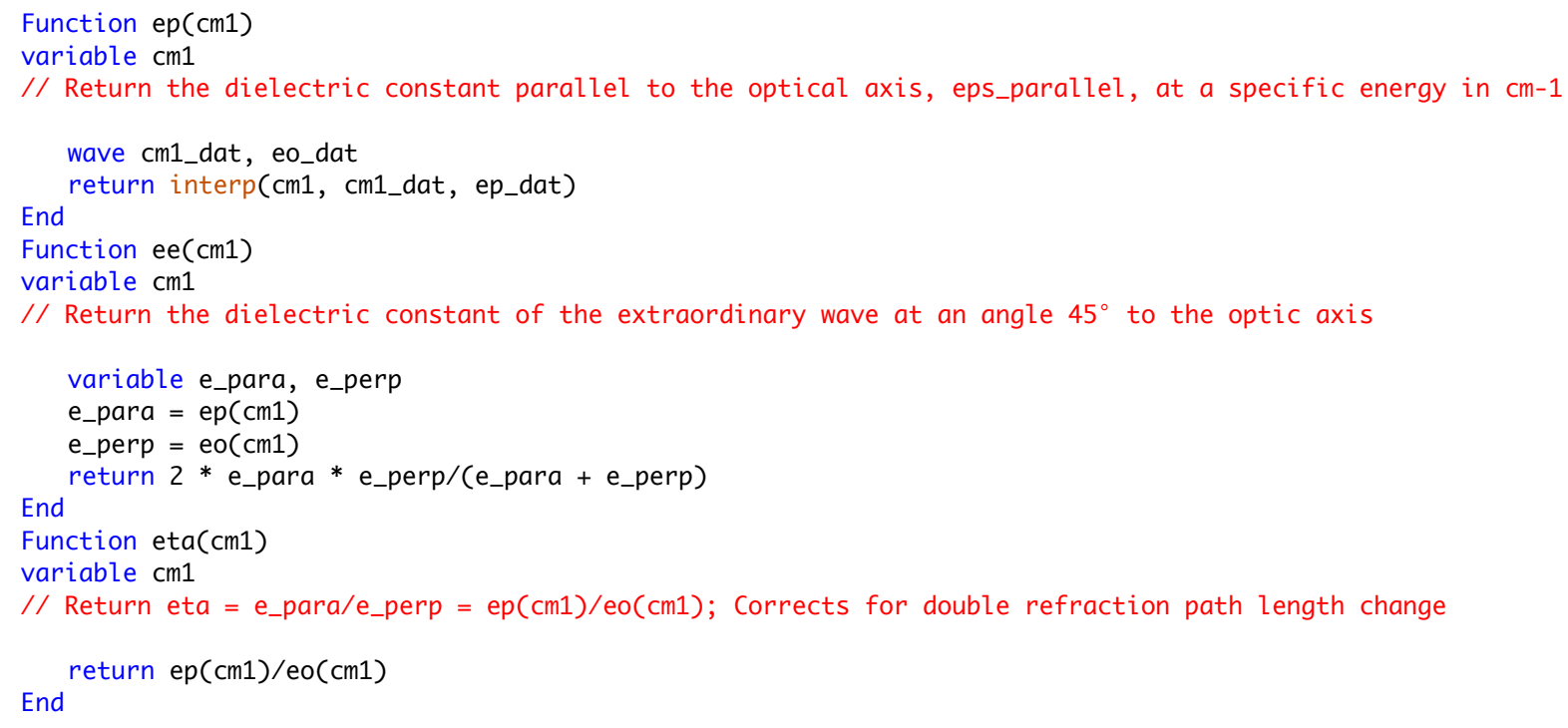

${ }^{1}$ Wagner, C. D.; Davis, L. E.; Zeller, M. V.; Taylor, J. A.; Raymond, R. H.; Gale, L. H. Empirical Atomic Sensitivity Factors for Quantitative Analysis By Electron Spectroscopy for Chemical Analysis. Surf. Interface Anal. 1981, 3, 211-225.

${ }^{2}$ Fuentes, G. G.; Elizalde, E.; Yubero, F.; Sanz, J. M. Electron Inelastic Mean Free Path for Ti, TiC, TiN, and $\mathrm{TiO}_{2}$ as Determined By Quantitative Reflection Electron Energy-Loss Spectroscopy. Surf. Interface Anal. 2002, 33, 230-237.

${ }^{3}$ WaveMetrics, Inc., https://www.wavemetrics.com/products/igorpro/igorpro.htm 